\title{
Subjective Sleep Quality in Amnestic Mild Cognitive Impairment Elderly and Its Possible Relationship With Plasma Amyloid- $\beta$
}

Yajing Liu't, Lushi Chen'1t, Shuyun Huang ${ }^{1}$, Chengguo Zhang1, Zeping Lv², Jiali Luo', Pan Shang ${ }^{1}$, Yukai Wang ${ }^{1 *}$ and Haiqun Xie ${ }^{1 *}$

${ }^{1}$ Department of Neurology, First People's Hospital of Foshan, Foshan, China, ${ }^{2}$ National Research Center for Rehabilitation Technical Aids, Rehabilitation Hospital, Beijing, China

Study objectives: To investigate the extent to which sleep quality associated with plasma A $\beta$ levels in amnestic mild cognitive impairment (aMCl) elderly.

OPEN ACCESS

Edited by:

Andrea Romigi,

Istituto Neurologico Mediterraneo Neuromed (IRCCS), Italy

Reviewed by: Michelangelo Maestri, University of Pisa, Italy Francesca Izzi,

Policlinico Tor Vergata, Italy

*Correspondence:

Yukai Wang

wykai2012@qq.com

Haiqun Xie

haiqunx@foxmail.com

tThese authors share first authorship

Specialty section: This article was submitted to Sleep and Circadian Rhythms, a section of the journal

Frontiers in Neuroscience

Received: 29 September 2020 Accepted: 23 November 2020 Published: 21 December 2020

Citation:

Liu Y, Chen L, Huang S, Zhang C, Lv Z, Luo J, Shang P, Wang Y and Xie H (2020) Subjective Sleep Quality

in Amnestic Mild Cognitive Impairment Elderly and Its Possible Relationship With Plasma Amyloid- $\beta$.

Front. Neurosci. 14:611432.

doi: 10.3389/fnins.2020.611432
Methods: A total of 172 cognitively normal (NC) elderly and $133 \mathrm{aMCl}$ elderly were included in this study. For the evaluation of sleep quality, the Pittsburgh Sleep Quality Index (PSQl) was used. Levels of plasma A $\beta$ were determined by the sandwich enzymelinked immunosorbent assay technique. Multivariable linear regression analysis was applied to evaluate associations between sleep quality and plasma $A \beta$ levels after adjusting potential confounders.

Results: Compared to NC subjects, participants with aMCI had a higher global PSQI score $(8.72 \pm 3.87$ vs. $7.10 \pm 3.07, p<0.001)$. The global PSQI score was positively associated with plasma $A \beta_{42}$ level in the aMCl group $(\beta=0.063,95 \% \mathrm{Cl} 0.001-0.125$, and $p=0.049$ ) but not in the NC group $(p>0.05)$. Additionally, a higher global PSQI score was associated with a higher plasma $A \beta_{42} / A \beta_{40}$ ratio in both $N C(\beta=0.010,95 \%$ $\mathrm{Cl} 0.003-0.016$, and $p=0.003)$ and aMCl groups $(\beta=0.012,95 \% \mathrm{Cl} 0.005-0.018$, and $p<0.001)$. The association between global PSQI score and plasma $A \beta_{42} / A \beta_{40}$ ratio was stronger in individuals with aMCl relative to the $\mathrm{NC}$ subjects $(\beta=0.076$ vs. 0.030, $p$ for interaction $=0.023$ ).

Conclusion: Poor sleep quality was associated with plasma $A \beta_{42}$ and $A \beta_{42} / A \beta_{40}$ ratio, with a stronger effect among individuals with $\mathrm{aMCl}$. A better understanding of the role of sleep in plasma $A \beta$ levels in aMCl patients could lead to effective sleep-based intervention against the risk of Alzheimer's disease.

Keywords: Alzheimer's disease, amnestic mild cognitive impairment, Pittsburgh Sleep Quality Index, sleep quality, plasma amyloid- $\beta$

\section{INTRODUCTION}

Sleep disorders are commonly seen in older people and increase with age (Gadie et al., 2017). Growing evidences suggested that poor sleep quality tends to impair cognitive function (Karatsoreos et al., 2011; Yaffe et al., 2011; Kwon et al., 2015). A cohort study with 15,246 older people found that low sleep efficiency was associated with a high risk of memory 
impairment and poor cognitive function (Ma et al., 2019). Some case-control studies indicated that compared to cognitively healthy elderly, patients with mild cognitive impairment (MCI) had a higher prevalence of sleep disorder (Chiu et al., 2016; Palmer et al., 2018; Carnicelli et al., 2019).

Sleep disorder has been recognized as a significant risk factor for the development of Alzheimer's disease (AD), which was attributed to amyloid- $\beta$ (A $\beta)$ deposition in the brain. Recent investigations verified that sleep/wake patterns might regulate $A \beta$ peptide. Sleep decreased $A \beta$ production and increased clearance, while wakefulness leads to an increase of $A \beta$ production in the brain (Slats et al., 2013; Xie et al., 2013). These underlying associations were confirmed by several studies. Ooms et al. (2014) found that sleep deprivation enhanced cerebrospinal fluid (CSF) $\mathrm{A} \beta$ levels. Compared to sleep control, sleep deprivation increased overnight CSF A $\beta$ levels by $25-30 \%$ via increased overnight $A \beta$ production (Lucey et al., 2018). Furthermore, sleep deprivation promoted amyloid plaque formation in both humans and rodents (Kang et al., 2009; Ooms et al., 2014; Lucey et al., 2018; Shokri-Kojori et al., 2018). A prospective study with 70 cognitively healthy adults showed that poor sleep quality was associated with an increased $A \beta$ burden in the brain (Spira et al., 2013).

The association between sleep quality and plasma $A \beta$ has recently gained more interest. It has been confirmed that plasma $\mathrm{A} \beta$ levels correlated closely to the brain $\mathrm{A} \beta$ burden (Tzen et al., 2014; Nakamura et al., 2018). A $\beta$ peptides involved dynamic interchange between the brain and periphery via transportation across the blood-brain barrier and blood-CSF barrier (Tarasoff-Conway et al., 2015). In light of these findings, few studies explored the association between sleep and plasma $\mathrm{A} \beta$. Grimmer et al. (2020) reported that plasma $\mathrm{A} \beta$ levels decreased significantly after overnight sleep, and this reduction could be diminished by sleep disturbance in healthy adults. Wei et al. (2017) observed that after total sleep deprivation, plasma $A \beta$ levels increased significantly in healthy young adults. A case-control study with 21 MCI patients suggested that disrupted slow-wave sleep was associated with plasma A $\beta$ levels (Sanchez-Espinosa et al., 2014).

Despite these evidences, distinct sleep disorder patterns have not yet been well understood, especially in subjects with amnestic mild cognitive impairment (aMCI), and a subtype of MCI with a high risk of developing AD (Albert et al., 2011). Moreover, how sleep quality during a period of time influences plasma $A \beta$ levels in aMCI individuals remains unclear. Understanding the effect of sleep disorder on aMCI may lead to effective sleep-based interventions for $\mathrm{AD}$ prevention. Given the acceptability and cost-effectiveness, blood-based plasma $A \beta$ assay may be suitable for further investigating the causal roles of sleep disorder on $A \beta$ levels among the large-scale general population. Hence, in this study, we aimed to primarily investigate the sleep characteristics of aMCI different from cognitively normal (NC) elderly and secondly evaluate the association between sleep quality and plasma $A \beta$ in aMCI subjects.

\section{MATERIALS AND METHODS}

\section{Participants}

In total, this study recruited 305 participants (aged 60-85), including 133 aMCI patients (mean age $69.68 \pm 6.84$ ) from Cognitive Disorders Clinics in the First People's Hospital of Foshan and $172 \mathrm{NC}$ subjects (mean age $68.59 \pm 5.97$ ) from community volunteers.

The diagnosis of aMCI (Petersen, 2004) was according to the following criteria: (1) subjective cognitive impairment and confirmed by an informant; (2) preserved activities of daily living confirmed by a clinician's interviews; (3) multi-domain or a single-domain cognitive decline, and abnormal objective memory impairment determined by a cutoff of 1.5 standard deviations below education and age-matched specific norms by memory test; (4) global score of Clinical Dementia Rating $(\mathrm{CDR})=0.5$ (Morris, 1993); and (5) absence of dementia according to the criteria of Diagnostic and Statistical Manual of Mental Disorders, Fourth Edition, and revised (DSM-IV$\mathrm{R})$. The inclusion criteria for NC participants were as follows: (1) cognitively normal confirmed by neuropsychological testing; and (2) $\mathrm{CDR}=0$.

The exclusion criteria included any of the following situations: (1) neurologic disorder, such as cerebral infarction or hemorrhage histories, Parkinson's disease, epilepsy, or brain surgery; (2) autoimmune or peripheral vascular disease or cancer histories; (3) severe mood disorder (evaluated by the clinician according to the medical history and neuropsychological test); (4) obstructive sleep apnea; and (5) severe somatic disease, such as liver or kidney failure, and severe cardiopulmonary disease.

The ethics committee approved the research proposal in the First People's Hospital of Foshan Research Ethics Board. We obtained written informed consent from the participants at enrollment.

\section{Plasma A $\beta$ Assessment}

Blood samples $(7 \mathrm{ml})$ were collected in $\mathrm{K} 3$ Ethylenediaminetetraacetic acid (EDTA) tubes after overnight fasting. The blood was then centrifuged $(3,000 \mathrm{rpm}, 10 \mathrm{~min}$, and $4^{\circ} \mathrm{C}$ ), and the top layer containing plasma was moved into the Eppendorf tube. The plasma was stored at $-80^{\circ} \mathrm{C}$ in 90 min after venipuncture. We used the sandwich enzyme-linked immunosorbent assay (ELISA) technique to assay the levels of plasma $A \beta$ (Pesini et al., 2012). $A \beta_{40}$ and $A \beta_{42}$ in plasma were assayed using Human/Rat $\beta$ Amyloid (40/42) ELISA Kit (Wako, Japan). Briefly, $100 \mu \mathrm{l}$ of standards and plasma samples were added, which were then incubated with plate seal overnight at $4^{\circ} \mathrm{C}$. One hundred microliters of the HRP-conjugated Antibody Solution was added, and the plate was sealed for $1 \mathrm{~h}$ at $4^{\circ} \mathrm{C}$. Subsequently, $100 \mu \mathrm{l}$ of TMB Solution was added, and then the plate was sealed, and incubated at room temperature in the dark. After $30 \mathrm{~min}, 100 \mu \mathrm{l}$ of Stop Solution was added. The absorbance was read at $450 \mathrm{~nm}$ with a microplate reader. We used synthetic $A \beta_{40}$ and $A \beta_{42}$ peptides to establish standard curves, and we determined the concentration of $A \beta$. 


\section{Sleep Assessment}

The Pittsburgh Sleep Quality Index (PSQI) was used to assess sleep quality (Buysse et al., 1989). All of the participants completed the PSQI on the day of blood collection and confirmed that the quality of nighttime sleep prior to sampling was consistent with the description in the scale, with no significant fluctuation. PSQI is a recognized, self-administered questionnaire. It comprises 19 items that reflect subjective sleep quality, sleep latency, sleep duration, sleep efficiency, sleep disturbance, daytime dysfunction, and the use of sleeping medication. The scores of these seven components were reassigned, each weighted equally on the 0-3 scale. Scores of seven components were then summed to a yield a global PSQI score (0-21 scale), with higher scores indicating worse sleep quality.

\section{Medical and Cognitive Assessment}

Data on demographic characteristics, medical history, lifestyle, physical measurements, and biochemical blood measurements were collected during health examinations. Participants completed a neuropsychological test battery, including MiniMental State Examination (MMSE) and the Chinese version of Montreal Cognitive Assessment (MoCA) to assess global cognition. Additionally, the Stroop's Color Word Test (SCWT) was used to evaluate the executive function, and the Boston Naming Test was used to determine the language. Then, the Symbol Digit Modalities Test (SDMT) was used to assess attention; the Auditory Verbal Learning Test-Huashan version (AVLT-H) was used to evaluate memory. Furthermore, the geriatric depression scale (GDS) score was collected to adjust for the effects of subsyndromal depression.

\section{Statistical Analysis}

The ratio of $A \beta_{42} / A \beta_{40}$ was log-transformed to correct skewness. The characteristics were compared between groups using Student's $t$ test for continuous variables and the $\chi^{2}$ test or Fisher's exact test for the categorical variables. Multivariable linear regression analysis was used to estimate the effect values $(\beta)$ and $95 \%$ confidence intervals (CIs) to examine the extent to which sleep quality associated with plasma $A \beta$ variables. Three different models were tested: an unadjusted model, Model I, and Model II. Model I was adjusted for age and gender. Model II additionally included apolipoprotein E (APOE) $\varepsilon 4$, education level, body mass index, exercise frequency, diabetes, hypertension, and the score of GDS. Sleep quality was stratified into tertile and then modeled as continuous variables to test for trends. Test for effect modification by the group employed

TABLE 1 | Characteristics of the study population.

\begin{tabular}{|c|c|c|c|c|}
\hline & Overall $(N=305)$ & $N C(N=172)$ & $\operatorname{aMCl}(N=133)$ & $p$ value \\
\hline Male, $n(\%)$ & $123(40.33)$ & 76 (44.19) & 47 (35.34) & 0.118 \\
\hline $\mathrm{A} \beta_{42}(\mathrm{pmol} / \mathrm{L})$ & $3.21 \pm 1.25$ & $3.06 \pm 1.20$ & $3.41 \pm 1.30$ & 0.013 \\
\hline$A \beta_{42} / A \beta_{40}{ }^{*}$ & $-1.09 \pm 0.14$ & $-1.11 \pm 0.12$ & $-1.06 \pm 0.14$ & $<0.001$ \\
\hline MMSE & $25.83 \pm 2.38$ & $26.91 \pm 1.73$ & $24.43 \pm 2.37$ & $<0.001$ \\
\hline $\mathrm{BMI}\left(\mathrm{kg} / \mathrm{m}^{2}\right)$ & $23.41 \pm 2.98$ & $23.66 \pm 2.86$ & $23.09 \pm 3.10$ & 0.105 \\
\hline $\mathrm{SBP}(\mathrm{mmHg})$ & $133.55 \pm 18.18$ & $133.65 \pm 18.43$ & $133.42 \pm 17.91$ & 0.917 \\
\hline $\mathrm{DBP}(\mathrm{mmHg})$ & $75.05 \pm 11.19$ & $75.84 \pm 11.47$ & $73.99 \pm 10.76$ & 0.165 \\
\hline FBS (mmol/L) & $5.20 \pm 1.20$ & $5.27 \pm 1.41$ & $5.12 \pm 0.83$ & 0.296 \\
\hline $\mathrm{TG}(\mathrm{mmol} / \mathrm{L})$ & $1.49 \pm 1.11$ & $1.56 \pm 1.35$ & $1.39 \pm 0.69$ & 0.192 \\
\hline $\mathrm{HBP}, n(\%)$ & 175 (57.95) & $99(57.56)$ & $76(58.46)$ & 0.875 \\
\hline $\mathrm{DM}, n(\%)$ & $42(13.77)$ & $24(13.95)$ & $18(13.53)$ & 0.916 \\
\hline Exercise, $n(\%)^{\&}$ & $220(72.13)$ & $126(73.26)$ & 94 (70.68) & 0.618 \\
\hline Education level (years) & & & & 0.003 \\
\hline $0, n(\%)$ & $10(3.28)$ & $2(1.16)$ & $8(6.02)$ & \\
\hline $1-6, n(\%)$ & $90(29.51)$ & $42(24.42)$ & $48(36.09)$ & \\
\hline$\geq 7, n(\%)$ & $205(67.21)$ & $128(74.42)$ & $77(57.89)$ & \\
\hline
\end{tabular}

Data expressed as mean \pm standard deviation or percentage (\%).

\&Exercise more than two times per week.

*Variables were log-transformed in the analyses.

$A \beta$, amyloid- $\beta ; A \beta_{42} / A \beta_{40}$, the ratio of $A \beta_{42}-A \beta_{40} ; M M S E$, mini-mental state examination; MoCA, Montreal Cognitive Assessment; GDS, Geriatric Depression Scale; $B M I$, body mass index; SBP, systolic blood pressure; DBP, diastolic blood pressure; FBS, fasting blood glucose; TG, triglyceride; CH, total cholesterol; HDL, high-density lipoprotein; LDL, low-density lipoprotein; UA, uric acid; Cr, creatinine; $A P O E$, apolipoprotein E; HBP, hypertension; DM, diabetes. 
interaction terms. Analyses were conducted by the statistical software packages $\mathrm{R}^{1}$ (The $\mathrm{R}$ Foundation) and Empower Stats ${ }^{2}$ (X\&Y solutions, Inc., Boston, MA, United States). $p$ value $<0.05$ was considered statistically significant.

\section{RESULTS}

The descriptive characteristics of the study population is shown in Table 1. Compared to NC (aged 60-85), participants with aMCI (aged 61-85) had lower levels of education $(p=0.003)$ and lower total scores of MMSE $(24.43 \pm 2.37$ vs. $26.91 \pm 1.73$, $p<0.001)$ and MoCA $(19.75 \pm 3.38$ vs. $23.74 \pm 2.89, p<0.001)$. Additionally, plasma $\mathrm{A} \beta_{42}$ level $(3.41 \pm 1.30$ vs. $3.06 \pm 1.20$, $p=0.013)$ and $\mathrm{A} \beta_{42} / \mathrm{A} \beta_{40}$ ratio $(-1.06 \pm 0.14$ vs. $-1.11 \pm 0.12$, $p<0.001)$ in participants with aMCI were higher than those in the NC group. Plasma $A \beta_{40}$ level, the GDS score, body mass index, systolic blood pressure, diastolic blood pressure, fasting blood glucose, triglyceride, total cholesterol, high-density lipoprotein, low-density lipoprotein, APOE $\varepsilon 4$, hypertension, diabetes, and exercise frequency did not differ between NC and aMCI groups $(p>0.05)$.

Table 2 shows the sleep characteristics of the aMCI and NC subjects. Compared to NC subjects, aMCI patients had higher global PSQI scores ( $8.72 \pm 3.87$ vs. $7.10 \pm 3.07, p<0.001)$, which suggested worse sleep quality. It mainly reflected worse subjective sleep quality, long sleep latency, short sleep duration, low sleep efficiency, and more frequent sleep disturbances.

Table 3 shows the association between the global PSQI score and plasma $A \beta$. The global PSQI score was positively associated with plasma $A \beta_{42}$ level in the aMCI group $(\beta=0.063,95 \% \mathrm{CI}$ $0.001-0.125$, and $p=0.049)$ but not in the NC group $(p>0.05)$. After stratifying the global PSQI score into tertile, compared to those in tertile 1 (score $<5$ ), participants in tertiles $2-3$ had a higher plasma $A \beta_{42}$ level in the aMCI group (tertile 2: $\beta=0.652$, 95\% CI 0.020-1.284, and $p=0.046$; tertile 3: $\beta=1.093$, 95\% CI $0.461-1.724$, and $p<0.001)$. We further tested the trends and found that the global PSQI score was positively associated with plasma $\mathrm{A} \beta_{42}$ level in the aMCI group $(p<0.001)$. Additionally, a high global PSQI score was associated with a high plasma $\mathrm{A} \beta_{42} / \mathrm{A} \beta_{40}$ ratio in both $\mathrm{NC}(\beta=0.010,95 \%$ CI $0.003-0.016$, and $p=0.003)$ and aMCI groups $(\beta=0.012$, 95\% CI $0.005-0.018$, and $p<0.001$ ). Compared to those with low score (tertile 1 ), participants with a high score (tertiles 2-3) had higher plasma $\mathrm{A} \beta_{42} / \mathrm{A} \beta_{40}$ ratio in both NC (tertile $2: \beta=0.046,95 \%$ CI $0.000-$ 0.093 , and $p=0.046$; tertile 3 : $\beta=0.060$, 95\% CI $0.015-0.106$, and $p=0.010$ ) and aMCI groups (tertile $2: \beta=0.109,95 \%$ CI $0.042-0.176$, and $p=0.002$; tertile $3: \beta=0.161,95 \% \mathrm{CI}$ $0.094-0.228$, and $p<0.001)$. After adjusting for confounders and potential mediators, these associations also persisted. The further test of trend showed that global PSQI score was positively associated with plasma $\mathrm{A} \beta_{42} / \mathrm{A} \beta_{40}$ ratio in both $\mathrm{NC}(\beta=0.030$, 95\% CI $0.008-0.053$, and $p=0.010)$ and aMCI $(\beta=0.076,95 \%$ CI $0.044-0.109$, and $p<0.001)$ groups. Moreover, interaction

${ }^{1}$ http://www.R-project.org

${ }^{2}$ www.empowerstats.com
TABLE 2 | Sleep quality of the study population.

\begin{tabular}{|c|c|c|c|}
\hline Sleep variable & $N C(N=172)$ & $\operatorname{aMCl}(N=133)$ & $p$ value \\
\hline Subjective sleep quality & & & 0.003 \\
\hline Very good, $n(\%)$ & $31(18.13)$ & $12(9.09)$ & \\
\hline Fairly good, $n(\%)$ & $96(55.56)$ & 71 (53.03) & \\
\hline Fairly bad, $n(\%)$ & $44(25.73)$ & $41(31.06)$ & \\
\hline Very bad, $n(\%)$ & $1(0.58)$ & $9(6.82)$ & \\
\hline Sleep latency & & & 0.006 \\
\hline$\leq 15 \min , n(\%)$ & $69(40.12)$ & $39(29.55)$ & \\
\hline $16-30 \min , n(\%)$ & $76(44.19)$ & $52(38.64)$ & \\
\hline $31-60$ min, $n(\%)$ & $21(12.21)$ & $28(21.21)$ & \\
\hline$\geq 60 \min , n(\%)$ & $6(3.49)$ & $14(10.61)$ & \\
\hline Sleep duration & & & 0.001 \\
\hline$>7 \mathrm{~h}, n(\%)$ & $37(21.51)$ & $17(12.78)$ & \\
\hline $6-7$ h, $n(\%)$ & $80(46.51)$ & $53(39.85)$ & \\
\hline 5-6 h, n (\%) & $51(29.65)$ & 47 (35.34) & \\
\hline$<5 \mathrm{~h}, n(\%)$ & $4(2.33)$ & $16(12.03)$ & \\
\hline Sleep efficiency & $78.35 \pm 10.82 \%$ & $73.06 \pm 12.08 \%$ & $<0.001$ \\
\hline$>85 \%, n(\%)$ & 39 (22.67) & 19 (14.39) & \\
\hline $75-84 \%, n(\%)$ & 68 (39.53) & 38 (28.79) & \\
\hline $65-74 \%, n(\%)$ & $47(27.33)$ & $40(29.55)$ & \\
\hline$<65 \%, n(\%)$ & $18(10.47)$ & $36(27.27)$ & \\
\hline Sleep disturbances & & & 0.047 \\
\hline Not at all, $n(\%)$ & $5(2.91)$ & $0(0.00)$ & \\
\hline At least once per week, $n(\%)$ & $167(97.09)$ & $133(100.00)$ & \\
\hline Daytime dysfunction & & & 0.994 \\
\hline Not at all, $n(\%)$ & $80(46.78)$ & $59(46.83)$ & \\
\hline At least once per week, $n(\%)$ & $92(53.22)$ & $67(53.17)$ & \\
\hline Sleeping medication & & & 0.558 \\
\hline Not at all, $n(\%)$ & $147(85.47)$ & $116(87.72)$ & \\
\hline Used, $n(\%)$ & $25(14.53)$ & $17(12.78)$ & \\
\hline Global PSQI score & $7.10 \pm 3.07$ & $8.72 \pm 3.87$ & $<0.001$ \\
\hline $\mathrm{T} 1(1-5)^{\#}, n(\%)$ & $61(35.47)$ & $26(19.55)$ & \\
\hline T2 (6-8), n (\%) & $50(29.07)$ & 46 (34.59) & \\
\hline T3 (9-19), n (\%) & $61(35.47)$ & $61(45.86)$ & \\
\hline
\end{tabular}

Pittsburgh Sleep Quality Index (PSQI) consisted of subjective sleep quality, sleep latency, sleep duration, sleep efficiency, sleep disturbance, daytime function, and use of sleeping medication. The scores of these seven components were reassigned, each weighted equally on $0-3$ scale.

Data expressed as mean \pm standard deviation or percentage (\%).

\# The low tertile of the global PSQ/ score, range of 1-5 scale.

terms were used to test effect modification by group classification (aMCI vs. NC). Patients with aMCI had a stronger association of global PSQI score (tertile) with plasma $A \beta_{42}$ level and $A \beta_{42} / A \beta_{40}$ ratio relative to the $\mathrm{NC}$ subjects $(\beta=0.076$ vs. $0.030, p$ for interaction $=0.023)$. There was no association between global PSQI score and plasma $\mathrm{A} \beta_{40}$ level in both NC and aMCI groups, even after adjusting for confounders and potential mediators (data not shown).

\section{DISCUSSION}

The present study evaluated the extent to which sleep quality was associated with plasma A $\beta$ levels in aMCI elderly. Poor sleep quality, as reflected by a high global PSQI score, was associated 
TABLE 3 | Association between sleep quality and plasma A $\beta$ levels.

\begin{tabular}{|c|c|c|c|}
\hline \multirow[t]{2}{*}{ Variable } & \multirow{2}{*}{$\frac{\text { Unadjusted model }}{(\text { Frame1) } \beta(95 \% \mathrm{Cl}) p \text { value }}$} & \multirow{2}{*}{$\frac{\text { Model I }}{(\text { Frame2) } \beta(95 \% \mathrm{Cl}) p \text { value }}$} & \multirow{2}{*}{$\frac{\text { Model II }}{(\text { Frame3) } \beta(95 \% \mathrm{Cl}) p \text { value }}$} \\
\hline & & & \\
\hline \multicolumn{4}{|l|}{$\begin{array}{c}A \beta_{42} \\
N C\end{array}$} \\
\hline Global PSQI score & $0.021(-0.037,0.079) 0.484$ & $0.025(-0.035,0.084) 0.419$ & $0.029(-0.031,0.089) 0.348$ \\
\hline $\mathrm{T} 1(1-5)^{\#}$ & Reference & Reference & Reference \\
\hline$p$ for trend & $0.067(-0.146,0.280) 0.539$ & $0.080(-0.135,0.296) 0.465$ & $0.089(-0.132,0.309) 0.432$ \\
\hline \multicolumn{4}{|l|}{ aMCl } \\
\hline Global PSQI score & $0.059(0.003,0.115) 0.042$ & $0.061(0.003,0.118) 0.041$ & $0.063(0.001,0.125) 0.049$ \\
\hline $\mathrm{T} 1(1-5)$ & Reference & Reference & Reference \\
\hline T2 (6-8) & $0.658(0.061,1.255) 0.033$ & $0.669(0.066,1.272) 0.031$ & $0.652(0.020,1.284) 0.046$ \\
\hline T3 (9-19) & $1.055(0.484,1.626)<0.001$ & $1.063(0.483,1.644)<0.001$ & $1.093(0.461,1.724)<0.001$ \\
\hline \multicolumn{4}{|l|}{ NC } \\
\hline Global PSQI score & $0.009(0.003,0.015) 0.003$ & $0.009(0.003,0.015) 0.003$ & $0.010(0.003,0.016) 0.003$ \\
\hline $\mathrm{T} 1(1-5)$ & Reference & Reference & Reference \\
\hline T2 (6-8) & $0.041(-0.005,0.087) 0.081$ & $0.041(-0.005,0.087) 0.061$ & $0.046(0.000,0.093) 0.046$ \\
\hline Т3 (9-19) & $0.058(0.014,0.101) 0.010$ & $0.059(0.015,0.103) 0.010$ & $0.060(0.015,0.106) 0.010$ \\
\hline$p$ for trend & $0.029(0.007,0.051) 0.010$ & $0.029(0.007,0.051) 0.009$ & $0.030(0.008,0.053) 0.010$ \\
\hline \multicolumn{4}{|l|}{$\mathrm{aMCl}$} \\
\hline Global PSQI score & $0.009(0.003,0.015) 0.004$ & $0.009(0.003,0.016) 0.004$ & $0.012(0.005,0.018)<0.001$ \\
\hline T1 (1-5) & Reference & Reference & Reference \\
\hline T2 (6-8) & $0.099(0.035,0.164) 0.003$ & $0.100(0.035,0.165) 0.003$ & $0.109(0.042,0.176) 0.002$ \\
\hline Т3 (9-19) & $0.138(0.076,0.200)<0.001$ & $0.141(0.078,0.203)<0.001$ & $0.161(0.094,0.228)<0.001$ \\
\hline
\end{tabular}

\# The low tertile of the global PSQ/ score, range of 1-5 scale.

*Variables were log-transformed in the analyses.

\&Interaction of group (aMCI vs. NC) on global PSQI score (tertile) and plasma AB.

$A \beta_{42} / A \beta_{40}$, the ratio of $A \beta_{42}-A \beta_{40} ; C l$, confidence interval.

Model I: adjusted for age and gender.

Model II: Model I plus adjusted for APOE\&4, education level, body mass index, exercise frequency, diabetes, hypertension, and geriatric depression scale score.

with increased plasma $\mathrm{A} \beta_{42}$ level in the aMCI group but not the NC group. A high $A \beta_{42} / A \beta_{40}$ ratio was associated with poor sleep quality, with a stronger effect among aMCI participants. Additionally, compared with normal control, aMCI subjects had worse sleep quality, indicated by worse subjective sleep quality, shorter sleep duration, longer sleep latency, lower sleep efficiency, and more sleep disturbances.

Growing evidence suggested that individuals with cognitive impairment had poor sleep quality relative to cognitively normal subjects. An interview survey with 2,413 elderlies in Taiwan reported that participants with cognitive impairment had a higher prevalence of self-reported sleep disturbances (Chiu et al., 2016). Palmer et al. (2018) showed that participants with MCI had a 3.2 higher odds of poor sleep measured by Sleep Continuity in Alzheimer's disease Scale. Recent studies showed that MCI patients had a disrupted sleep with decreased rapid eye movement sleep and cyclic alternating pattern rate (Carnicelli et al., 2019), as well as poorer spindle and K-complex activities (Liu et al., 2020). Results in our study were consistent with previous studies. Importantly, we further characterized patterns of sleep quality and provided more detailed sleep disorder patterns, including subjective sleep quality, sleep duration, sleep latency, sleep efficiency, as well as the prevalence of sleep disturbances, which were not previously reported for individuals with aMCI.

In this study, we revealed that poor sleep quality was associated with increased plasma $A \beta_{42}$ level in aMCI patients, as well as $A \beta_{42} / A \beta_{40}$ ratio in both NC and aMCI subjects. The underlying mechanism may be due to the following evidences. Firstly, the effect of sleep quality on $A \beta$ mainly correlated with neuronal activity and synaptic strength. During wakefulness, neuronal activity increases and releases soluble $\mathrm{A} \beta$. Conversely, during sleep, neuronal activity decreases, and $A \beta$ production reduces (Cirrito et al., 2005). Thus, it is conceivable that sleep could decrease $A \beta$ levels in the brain. Secondly, $A \beta$ peptides were interchanged dynamically between the brain and periphery (Tarasoff-Conway et al., 2015). In light of these findings, plasma A $\beta$ levels may be subject to sleep-wake states. Poor sleep 
quality, with an increased neuronal activity (Krueger et al., 2008; Vyazovskiy et al., 2009), might diminish physiological reduction of $A \beta$ and lead to a relatively high level of plasma $A \beta$.

The association between sleep quality and plasma $A \beta$ was different in $\mathrm{NC}$ and aMCI subjects. Our results for the first time to date suggested a high $A \beta_{42} / A \beta_{40}$ ratio, and increased $A \beta_{42}$ levels were associated with poor sleep quality, with a stronger effect among aMCI participants relative to NC elderly. The underlying mechanism may be explained by recent findings. The relationship between poor sleep and $A \beta$ accumulation is bidirectional (Ju et al., 2014). Poor sleep might increase $A \beta$ levels in the brain. In particular, $A \beta_{42}$ has long been recognized as a hydrophobic isoform with a tendency toward to form hard-to-clear aggregates (Tarasoff-Conway et al., 2015), which in turn increases the risk of amyloid plaque aggregation. Once amyloid plaques have formed, sleep-wake functions and circadian rhythms are disrupted (Roh et al., 2012; Ju et al., 2013). In comparison to healthy elderly, individuals with aMCI had a worse sleep quality in the present study. Additionally, previous evidence indicated that aMCI subjects had higher CSF $\mathrm{A} \beta_{42}$ levels, as well as more amyloid plaque formation than healthy elderly (Visser et al., 2009; Hanon et al., 2018; Knezevic et al., 2018). As a result, the bidirectional relationship between poor sleep and $A \beta_{42}$ levels in the brain was more significant in individuals with aMCI. Given that $\mathrm{A} \beta$ peptides dynamically interchanged between the brain and periphery, the extent to which poor sleep quality associated with high plasma $A \beta_{42}$ level and $A \beta_{42} / A \beta_{40}$ ratio in aMCI patients might be more significant than that in the NC subjects.

In this study, worse sleep quality was associated with a higher plasma $A \beta_{42} / A \beta_{40}$ ratio, but not $A \beta_{42}$ level in the NC subjects. It is possible that the concentration of $A \beta_{42}$ in plasma is much lower than that in CSF (Hanon et al., 2018). Evidence indicated that compared to the single peptide level in plasma, the ratio of $A \beta_{42} / A \beta_{40}$ had a higher sensitivity in predicting the $A \beta$ burden in the brain (Nakamura et al., 2018).

This study has limitations worth noting. Firstly, sleep quality was evaluated with PSQI, a self-report, which may be less objective than polysomnography. Secondly, participants who had suspected obstructive sleep apnea syndrome were ruled out based on the medical history, as well as on their response to the question "cough or snore loudly" or "cannot breathe comfortably" during sleep in the PSQI. PSQI is a measurement of sleep quality; however, the sensitivity and accuracy in identifying sleep apnea syndrome are worse than polysomnography. It is necessary to apply more scientific wearable sleep monitoring equipment to determine the relationship between sleep and plasma $A \beta$ in largesized cohorts in the future. Thirdly, we did not detect CSF $A \beta$ levels synchronously. Examining $A \beta$ level in both CSF and plasma may be better to understand the effect of sleep duration and efficiency on $A \beta$ metabolism, namely, how sleep disorder influences $A \beta$ production, and how $A \beta$ is cleared from the brain via the brain and periphery interchange pathway.

The present study provided evidence that poor sleep quality was associated with a high level of plasma $A \beta$ in no-demented elderly, especially aMCI patients. These findings have significant implications for the prevention strategies of $\mathrm{AD}$. As sleep quality can be intervened with drugs and physical activities, interventions to improve sleep quality may be conducive to delay the progression of $\mathrm{aMCI}$ to $\mathrm{AD}$, altering the risk of $\mathrm{AD}$ onset. Additionally, in comparison to PET imaging and CSF detection, plasma $A \beta$ assay is more cost-effective and less invasive, and thus might serve as an available tool for dynamic monitoring $A \beta$ levels. Thereby, our study provided a significant basis for using plasma $\mathrm{A} \beta$ as a convenient tool to monitor and evaluate the effectiveness of sleep-based interventions. Moreover, PSQI has demonstrated consistent internal reliability and construct validity for sleep evaluation (Spira et al., 2012). Assessing sleep quality with PSQI is more convenient and cost-effective, reflecting usual sleep habits and quality without interference with the environment, thus allowing for assessing sleep quality in large-sized cohorts. Our findings might be helpful to identify sleep disorder as a modifiable risk and predictive factor, as well as facilitate proposing early intervention strategies for $\mathrm{AD}$ prevention. Future studies would be more optimal by applying wearable sleep monitoring equipment to further explore the relationship between sleep and plasma $A \beta$.

\section{CONCLUSION}

These findings indicated that compared to $\mathrm{NC}$ subjects, aMCI patients had worse sleep quality. Furthermore, poor sleep quality was associated with plasma $A \beta_{42}$ and $A \beta_{42} / A \beta_{40}$ ratio, with a stronger effect among individuals with aMCI. A better understanding of the sleep characteristics and the role of sleep in plasma $A \beta$ levels in aMCI patients could lead to effective sleep-based intervention against the risk of $\mathrm{AD}$. Our findings might be helpful to identify modifiable risk and predictive factors and facilitate proposing early intervention strategies for $\mathrm{AD}$ prevention.

\section{DATA AVAILABILITY STATEMENT}

The raw data supporting the conclusions of this article will be made available by the authors, without undue reservation.

\section{ETHICS STATEMENT}

The studies involving human participants were reviewed and approved by First People's Hospital of Foshan Research Ethics Board. The patients/participants provided their written informed consent to participate in this study.

\section{AUTHOR CONTRIBUTIONS}

YL and LC contributed to the literature search, data analysis, and the draft of the manuscript. SH, CZ, and ZL contributed to the acquisition of data. JL and PS undertook laboratory detection. HX and YW contributed to the study design, study supervision, and the revisions of the manuscript. All authors contributed to the article and approved the submitted version. 


\section{FUNDING}

This work was supported by the National Key R\&D Program of China (Grant No. 2018YFC2001700) and the National Key Technology Research and Development Program of the Ministry of Science and Technology of China (Grant No. 2015BAI06B02).

\section{REFERENCES}

Albert, M. S., DeKosky, S. T., Dickson, D., Dubois, B., Feldman, H. H., Fox, N. C., et al. (2011). The diagnosis of mild cognitive impairment due to Alzheimer's disease: recommendations from the National Institute on Aging-Alzheimer's association workgroups on diagnostic guidelines for Alzheimer's disease. Alzheimers Dement. 7, 270-279. doi: 10.1016/j.jalz.2011.0 3.008

Buysse, D. J., Reynolds, C. R., Monk, T. H., Berman, S. R., and Kupfer, D. J. (1989). The pittsburgh sleep quality index: a new instrument for psychiatric practice and research. Psychiatry Res. 28, 193-213. doi: 10.1016/0165-1781(89)90 047-4

Carnicelli, L., Maestri, M., Di Coscio, E., Tognoni, G., Fabbrini, M., Schirru, A., et al. (2019). A longitudinal study of polysomnographic variables in patients with mild cognitive impairment converting to Alzheimer's disease. J. Sleep Res. 28:e12821. doi: 10.1111/jsr.12821

Chiu, H. Y., Lai, F. C., Chen, P. Y., and Tsai, P. S. (2016). Differences between men and women aged 65 and older in the relationship between Self-Reported sleep and cognitive impairment: a nationwide survey in taiwan. J. Am. Geriatr. Soc. 64, 2051-2058. doi: 10.1111/jgs.14316

Cirrito, J. R., Yamada, K. A., Finn, M. B., Sloviter, R. S., Bales, K. R., May, P. C., et al. (2005). Synaptic activity regulates interstitial fluid amyloid-beta levels in vivo. Neuron 48, 913-922. doi: 10.1016/j.neuron.2005.10.028

Gadie, A., Shafto, M., Leng, Y., and Kievit, R. A. (2017). How are agerelated differences in sleep quality associated with health outcomes? An epidemiological investigation in a UK cohort of 2406 adults. BMJ Open 7:e14920. doi: 10.1136/bmjopen-2016-014920

Grimmer, T., Laub, T., Hapfelmeier, A., Eisele, T., Fatke, B., Holzle, P., et al. (2020). The overnight reduction of amyloid beta 1-42 plasma levels is diminished by the extent of sleep fragmentation, sAPP-beta, and APOE epsilon4 in psychiatrists on call. Alzheimers Dement. 16, 759-769. doi: 10.1002/alz.12072

Hanon, O., Vidal, J. S., Lehmann, S., Bombois, S., Allinquant, B., Treluyer, J. M., et al. (2018). Plasma amyloid levels within the Alzheimer's process and correlations with central biomarkers. Alzheimers Dement. 14, 858-868. doi: 10.1016/j.jalz.2018.01.004

Ju, Y. E., Lucey, B. P., and Holtzman, D. M. (2014). Sleep and Alzheimer disease pathology-a bidirectional relationship. Nat. Rev. Neurol. 10, 115-119. doi: 10. 1038/nrneurol.2013.269

Ju, Y. E., McLeland, J. S., Toedebusch, C. D., Xiong, C., Fagan, A. M., Duntley, S. P., et al. (2013). Sleep quality and preclinical Alzheimer disease. JAMA Neurol. 70, 587-593. doi: 10.1001/jamaneurol.2013.2334

Kang, J. E., Lim, M. M., Bateman, R. J., Lee, J. J., Smyth, L. P., Cirrito, J. R., et al. (2009). Amyloid-beta dynamics are regulated by orexin and the sleep-wake cycle. Science 326, 1005-1007. doi: 10.1126/science.1180962

Karatsoreos, I. N., Bhagat, S., Bloss, E. B., Morrison, J. H., and McEwen, B. S. (2011). Disruption of circadian clocks has ramifications for metabolism, brain, and behavior. Proc. Natl. Acad. Sci. U.S.A. 108, 1657-1662. doi: 10.1073/pnas. 1018375108

Knezevic, D., Verhoeff, N., Hafizi, S., Strafella, A. P., Graff-Guerrero, A., Rajji, T., et al. (2018). Imaging microglial activation and amyloid burden in amnestic mild cognitive impairment. J. Cereb. Blood Flow Metab. 38, 1885-1895. doi: 10.1177/0271678X17741395

Krueger, J. M., Rector, D. M., Roy, S., Van Dongen, H. P., Belenky, G., and Panksepp, J. (2008). Sleep as a fundamental property of neuronal assemblies. Nat. Rev. Neurosci. 9, 910-919. doi: 10.1038/nrn2521

Kwon, K. J., Lee, E. J., Kim, M. K., Jeon, S. J., Choi, Y. Y., Shin, C. Y., et al. (2015). The potential role of melatonin on sleep deprivation-induced cognitive impairments: implication of FMRP on cognitive function. Neuroscience 301, 403-414. doi: 10.1016/j.neuroscience.2015.05.079

\section{ACKNOWLEDGMENTS}

We would like to thank Weiping Zhang and Biqing Lin for the screening tests in our clinic population. We would also like to thank Qiulan Huang for assistance in data collection and recruitment.

Liu, S., Pan, J., Tang, K., Lei, Q., He, L., Meng, Y., et al. (2020). Sleep spindles, $\mathrm{K}$-complexes, limb movements and sleep stage proportions may be biomarkers for amnestic mild cognitive impairment and Alzheimer's disease. Sleep Breath 24, 637-651. doi: 10.1007/s11325-019-01970-9

Lucey, B. P., Hicks, T. J., McLeland, J. S., Toedebusch, C. D., Boyd, J., Elbert, D. L., et al. (2018). Effect of sleep on overnight cerebrospinal fluid amyloid beta kinetics. Ann. Neurol. 83, 197-204. doi: 10.1002/ana.25117

Ma, X. Q., Jiang, C. Q., Xu, L., Zhang, W. S., Zhu, F., Jin, Y. L., et al. (2019). Sleep quality and cognitive impairment in older Chinese: Guangzhou Biobank Cohort Study. Age Ageing 49, 119-124. doi: 10.1093/ageing/afz120

Morris, J. C. (1993). The clinical dementia rating (CDR): current version and scoring rules. Neurology 43, 2412-2414. doi: 10.1212/wnl.43.11.2412-a

Nakamura, A., Kaneko, N., Villemagne, V. L., Kato, T., Doecke, J., Dore, V., et al. (2018). High performance plasma amyloid-beta biomarkers for Alzheimer's disease. Nature 554, 249-254. doi: 10.1038/nature25456

Ooms, S., Overeem, S., Besse, K., Rikkert, M. O., Verbeek, M., and Claassen, J. A. (2014). Effect of 1 night of total sleep deprivation on cerebrospinal fluid betaamyloid 42 in healthy middle-aged men: a randomized clinical trial. JAMA Neurol. 71, 971-977. doi: 10.1001/jamaneurol.2014.1173

Palmer, K., Mitolo, M., Burgio, F., Meneghello, F., and Venneri, A. (2018). Sleep disturbance in mild cognitive impairment and association with cognitive functioning. A Case-Control study. Front. Aging Neurosci. 10:360. doi: 10.3389/ fnagi.2018.00360

Pesini, P., Perez-Grijalba, V., Monleon, I., Boada, M., Tarraga, L., Martinez-Lage, P., et al. (2012). Reliable measurements of the beta-Amyloid pool in blood could help in the early diagnosis of AD. Int. J. Alzheimers Dis. 2012:604141. doi: 10.1155/2012/604141

Petersen, R. C. (2004). Mild cognitive impairment as a diagnostic entity. J. Intern. Med. 256, 183-194. doi: 10.1111/j.1365-2796.2004.01388.x

Roh, J. H., Huang, Y., Bero, A. W., Kasten, T., Stewart, F. R., Bateman, R. J., et al. (2012). Disruption of the sleep-wake cycle and diurnal fluctuation of beta-amyloid in mice with Alzheimer's disease pathology. Sci. Transl. Med. 4, 122r-150r. doi: 10.1126/scitranslmed.3004291

Sanchez-Espinosa, M. P., Atienza, M., and Cantero, J. L. (2014). Sleep deficits in mild cognitive impairment are related to increased levels of plasma amyloidbeta and cortical thinning. Neuroimage 98, 395-404. doi: 10.1016/j.neuroimage. 2014.05.027

Shokri-Kojori, E., Wang, G. J., Wiers, C. E., Demiral, S. B., Guo, M., Kim, S. W., et al. (2018). Beta-Amyloid accumulation in the human brain after one night of sleep deprivation. Proc. Natl. Acad. Sci. U.S.A. 115, 4483-4488. doi: 10.1073/ pnas. 1721694115

Slats, D., Claassen, J. A., Verbeek, M. M., and Overeem, S. (2013). Reciprocal interactions between sleep, circadian rhythms and Alzheimer's disease: focus on the role of hypocretin and melatonin. Ageing Res. Rev. 12, 188-200. doi: 10.1016/j.arr.2012.04.003

Spira, A. P., Beaudreau, S. A., Stone, K. L., Kezirian, E. J., Lui, L. Y., Redline, S., et al. (2012). Reliability and validity of the pittsburgh sleep quality index and the epworth sleepiness scale in older men. J. Gerontol. Ser. A Biol. Sci. Med. Sci. 67A, 433-439. doi: 10.1093/gerona/glr172

Spira, A. P., Gamaldo, A. A., An, Y., Wu, M. N., Simonsick, E. M., Bilgel, M., et al. (2013). Self-reported sleep and beta-amyloid deposition in communitydwelling older adults. JAMA Neurol. 70, 1537-1543. doi: 10.1001/jamaneurol. 2013.4258

Tarasoff-Conway, J. M., Carare, R. O., Osorio, R. S., Glodzik, L., Butler, T., Fieremans, E., et al. (2015). Clearance systems in the brain-implications for Alzheimer disease. Nat. Rev. Neurol. 11, 457-470. doi: 10.1038/nrneurol.2015. 119

Tzen, K. Y., Yang, S. Y., Chen, T. F., Cheng, T. W., Horng, H. E., Wen, H. P., et al. (2014). Plasma Abeta but not tau is related to brain PiB retention in 
early Alzheimer's disease. ACS Chem. Neurosci. 5, 830-836. doi: 10.1021/cn50 $0101 \mathrm{j}$

Visser, P. J., Verhey, F., Knol, D. L., Scheltens, P., Wahlund, L. O., Freund-Levi, Y., et al. (2009). Prevalence and prognostic value of CSF markers of Alzheimer's disease pathology in patients with subjective cognitive impairment or mild cognitive impairment in the DESCRIPA study: a prospective cohort study. Lancet Neurol. 8, 619-627. doi: 10.1016/S1474-4422(09)70139-5

Vyazovskiy, V. V., Olcese, U., Lazimy, Y. M., Faraguna, U., Esser, S. K., Williams, J. C., et al. (2009). Cortical firing and sleep homeostasis. Neuron 63, 865-878. doi: 10.1016/j.neuron.2009.08.024

Wei, M., Zhao, B., Huo, K., Deng, Y., Shang, S., Liu, J., et al. (2017). Sleep deprivation induced plasma amyloid-beta transport disturbance in healthy young adults. J. Alzheimers. Dis. 57, 899-906. doi: 10.3233/JAD-161213

Xie, L., Kang, H., Xu, Q., Chen, M. J., Liao, Y., Thiyagarajan, M., et al. (2013). Sleep drives metabolite clearance from the adult brain. Science 342, 373-377. doi: $10.1126 /$ science. 1241224
Yaffe, K., Laffan, A. M., Harrison, S. L., Redline, S., Spira, A. P., Ensrud, K. E. et al. (2011). Sleep-disordered breathing, hypoxia, and risk of mild cognitive impairment and dementia in older women. JAMA 306, 613-619. doi: 10.1001/ jama.2011.1115

Conflict of Interest: The authors declare that the research was conducted in the absence of any commercial or financial relationships that could be construed as a potential conflict of interest.

Copyright (c) 2020 Liu, Chen, Huang, Zhang, Lv, Luo, Shang, Wang and Xie. This is an open-access article distributed under the terms of the Creative Commons Attribution License (CC BY). The use, distribution or reproduction in other forums is permitted, provided the original author(s) and the copyright owner(s) are credited and that the original publication in this journal is cited, in accordance with accepted academic practice. No use, distribution or reproduction is permitted which does not comply with these terms. 